Physics

Physics Research Publications

\title{
Ultrasensitive mass sensing using mode localization in coupled microcantilevers
}
M. Spletzer
A. Raman
A. Q. Wu

X. F. Xu

R. Reifenberger

This paper is posted at Purdue e-Pubs.

http://docs.lib.purdue.edu/physics_articles/238 


\title{
Ultrasensitive mass sensing using mode localization in coupled microcantilevers
}

\author{
Matthew Spletzer, Arvind Raman, ${ }^{\text {a) }}$ Alexander Q. Wu, and Xianfan Xu \\ School of Mechanical Engineering and Birck Nanotechnology Center, Purdue University, West Lafayette, \\ Indiana 47907 \\ Ron Reifenberger \\ Department of Physics and Birck Nanotechnology Center, Purdue University, West Lafayette, \\ Indiana 47907
}

(Received 14 December 2005; accepted 23 May 2006; published online 20 June 2006)

\begin{abstract}
We use Anderson or vibration localization in coupled microcantilevers as an extremely sensitive method to detect the added mass of a target analyte. We focus on the resonance frequencies and eigenstates of two nearly identical coupled gold-foil microcantilevers. Theoretical and experimental results indicate that the relative changes in the eigenstates due to the added mass can be orders of magnitude greater than the relative changes in resonance frequencies. Moreover this sensing paradigm possesses intrinsic common mode rejection characteristics thus providing an alternate way to achieve ultrasensitive mass detection under ambient conditions. (C) 2006 American Institute of Physics. [DOI: 10.1063/1.2216889]
\end{abstract}

Microcantilever gravimetric sensors have emerged over the past decade as a promising technology for rapid and sensitive biochemical analyte detection. The added mass of the target analyte, which binds to cantilever coatings due to molecule specific interactions, is detected as a decrease in the cantilever resonance frequency. Vapors, ${ }^{1,2}$ trinitrotoluene (TNT) deflagration, ${ }^{3}$ and individual virus particles ${ }^{4}$ have been detected using this transduction mechanism. The most sensitive microcantilever based mass detection experiments using the frequency shift approach have reported attogram level detection in ultrahigh vacuum environments ${ }^{5-7}$ and femtogram level detection under ambient conditions. ${ }^{4,8,9}$

In what follows, we propose the use of Anderson or mode localization in the vibrations of two nearly identical coupled cantilevers as a means for improving by orders of magnitude the sensitivity of micromechanical mass detection compared to the conventional frequency shift approach. The vibrations of this mechanical system bear an elegant analogy to a quantum mechanical system, namely, the hydrogen molecule. Like the wave functions of a hydrogen molecule, the eigenstates of two coupled identical microcantilevers can be classified as symmetric (bonding orbital) and antisymmetric (antibonding orbital). Moreover the resonance frequency (energy state) of the antisymmetric eigenstate is greater than that of the symmetric eigenstate. Whereas the electrostatic potential well of the hydrogen molecule is inherently symmetric, the mechanical analog permits the selective introduction of disorder into the eigenvalue problem, for example, by means of the added mass of a target analyte. We show in this letter that the shifts in eigenstates of this mechanical analog of the hydrogen molecule due to an added mass can be orders of magnitude greater than the shifts in resonance frequency.

While numerous studies of mode localization in coupled structures $^{10-15}$ and arrays of coupled oscillators ${ }^{16-18}$ have been performed, the question of whether this phenomenon can be used in a sensing capacity has not been examined.

\footnotetext{
${ }^{a)}$ Electronic mail: raman@ecn.purdue.edu
}

This question is addressed in this letter both theoretically and by means of experiments using coupled gold-foil microcantilevers.

A schematic of two identical cantilevers coupled by means of an overhang is shown in Fig. 1(a). In order to understand the underlying physics of this system it is sufficient to use a discretized model shown in Fig. 1(b). Each cantilever is modeled as a damped simple harmonic oscillator, while the effect of the overhang coupling is modeled as a spring connecting the two oscillators. $K_{1}, M_{1}$ and $K_{2}, M_{2}$ are, respectively the bending stiffness and suspended mass of the two cantilevers, while $\Delta M$ is the effective mass of the analyte being detected. $K_{c}$ is the stiffness of the overhang coupling the two cantilevers.

Consider first the case of two initially identical cantilevers, $K_{1}=K_{2}=K, M_{1}=M_{2}=M$. The eigenvalue problem governing the undamped free oscillations of the system [taking $\left[\begin{array}{ll}x_{1} & x_{2}\end{array}\right]^{T}=\boldsymbol{u} e^{j \lambda t}$ in Fig. 1(b) $]$ can then be nondimensionalized and written as follows:

$$
\left[\begin{array}{cc}
1+\kappa & -\kappa \\
-\kappa & (1+\kappa) /(1+\delta)
\end{array}\right] \boldsymbol{u}=\lambda \boldsymbol{u},
$$

where $\delta$ represents the ratio of the effective mass added to the second cantilever to the single cantilever mass $\Delta M / M$, and $\kappa>0$ represents the ratio of the coupling stiffness to the cantilever stiffness, $K_{C} / K$. It can be shown that if $\delta=0$, the

(a)
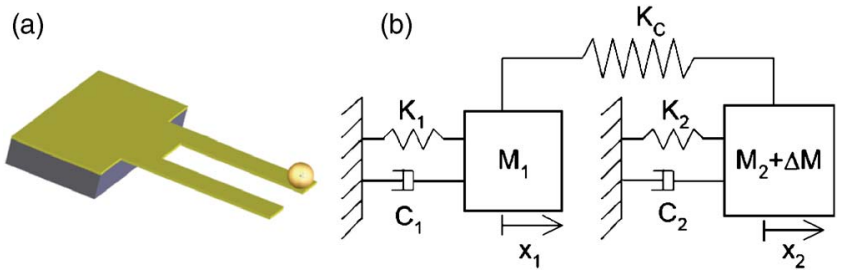

FIG. 1. (Color online) (a) Schematic of the nominal dimensions of the coupled gold-foil cantilevers fabricated and analyzed in this work with a sphere representing the mass placed at the end of one cantilever and (b) simplified model of the coupled gold-foil cantilevers. 
eigenvalues and normalized eigenstates of the coupled system are

$$
\lambda_{1}^{0}=1, \quad \boldsymbol{u}_{1}^{0}=\frac{1}{\sqrt{2}}\left[\begin{array}{l}
1 \\
1
\end{array}\right], \quad \lambda_{2}^{0}=2 \kappa+1, \quad \boldsymbol{u}_{2}^{0}=\frac{1}{\sqrt{2}}\left[\begin{array}{c}
1 \\
-1
\end{array}\right] .
$$

The superscript 0 will be used in subsequent expressions to denote eigenvalues and eigenstate of the system when $\delta=0$. The lower eigenvalue $\lambda_{1}^{0}$ corresponds to a symmetric eigenstate $\boldsymbol{u}_{1}^{0}$ where both masses oscillate in phase, while the higher eigenvalue $\lambda_{2}^{0}$ corresponds to an antisymmetric eigenstate $\boldsymbol{u}_{2}^{0}$ where the two masses oscillate out of phase. Both these eigenstates are said to be nonlocalized because the magnitudes of the displacements of each mass in either of the two modes are equal. This is the exact mechanical analog of the wave functions of the hydrogen molecule.

When $\delta \neq 0$, the added mass breaks the symmetry of the eigenvalue problem. It is convenient to expand the resulting eigenvalues $\lambda_{i}, i=1,2$ and eigenstates $\boldsymbol{u}_{i}, i=1,2$ in a perturbation expansion in $\delta$ (so long as $\delta \ll \kappa$ ) using the eigenvalue perturbation method of Courant and Hilbert. ${ }^{19}$ Accordingly,

$$
\lambda_{i}=\lambda_{i}^{0}+\delta \lambda_{i}^{1}+O\left(\delta^{2}\right), \quad \boldsymbol{u}_{i}=\boldsymbol{u}_{i}^{0}+\delta \boldsymbol{u}_{i}^{1}+O\left(\delta^{2}\right), \quad i=1,2 .
$$

Equation (3) can be substituted into Eq. (1) and the resulting eigenvalue problem can be solved at each order of perturbation given the condition that each eigenstate is normalized $\left(\boldsymbol{u}_{i}^{T} \boldsymbol{u}_{i}=1, i=1,2\right)$. From this analysis it can be seen that the relative change in normalized eigenstate is given by

$$
\frac{\left|\boldsymbol{u}_{i}-\boldsymbol{u}_{i}^{0}\right|}{\left|\boldsymbol{u}_{i}^{0}\right|}=\left(\frac{1}{4}+\frac{1}{4 \kappa}\right) \delta, \quad i=1,2,
$$

while the relative change in the eigenvalue or resonance frequency of a single cantilever is given by

$$
\frac{\lambda-\lambda_{0}}{\lambda_{0}}=\frac{-\delta}{2} .
$$

Note that the perturbed eigenstates $\boldsymbol{u}_{i}, i=1,2$ start becoming localized in the sense that in each eigenstate one cantilever oscillates more than the other.

Equation (4), which defines the sensed quantity in this sensing paradigm, suggests that simply by decreasing the scaled coupling between the two cantilevers $\kappa$, the relative changes in eigenstates can be made orders of magnitude greater than the relative change in eigenvalue of a single cantilever. In the case of the coupled gold-foil cantilevers fabricated in this work, we find that $\kappa \sim 10^{-2}$. This implies that the relative change in eigenstates of the coupled cantilevers in this work can be two orders of magnitude greater than the relative change in resonance frequencies.

Ideally, the cantilevers are identical; however, manufacturing tolerances and material variations cause the properties of one cantilever to differ slightly from another, introducing initial disorder into each set of coupled gold-foil cantilevers. In order to examine the effects of such initial disorder, hundreds of simulations are performed in which the dimensions of both cantilevers are varied randomly about the nominal value of the fabricated sensors. ${ }^{20} \mathrm{We}$ find that the presence of initial disorder can decrease or increase the sensitivity of the system slightly; however, the average relative change in the Downloaded 27 Jul 2006 to 128.210.146.185. Redistribution subject
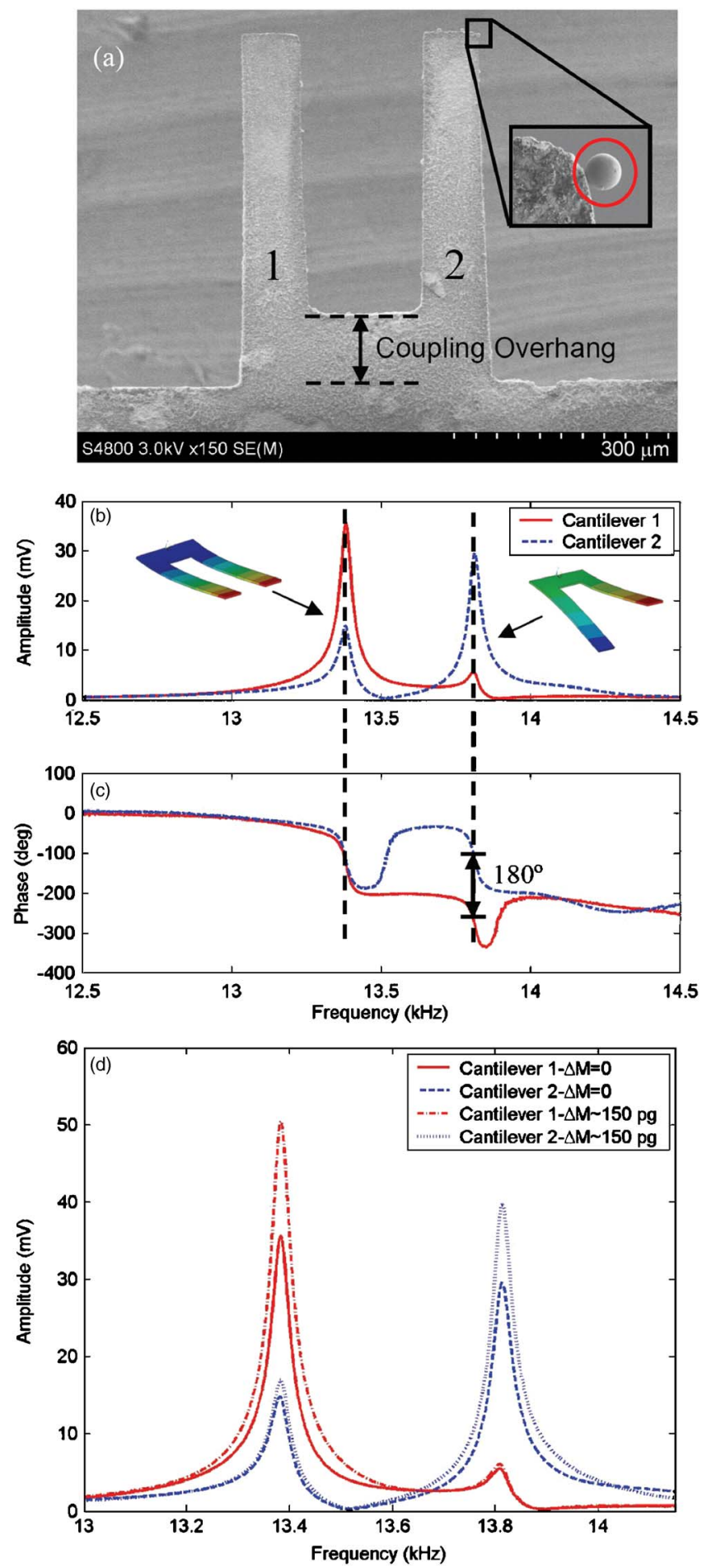

FIG. 2. (Color online) (a) scanning electromicroscopy (SEM) image of the first set of coupled gold-foil cantilevers and SEM image of an attached microsphere (inset, circled). (b) Amplitude vs frequency and (c) phase vs frequency of the coupled gold-foil cantilevers. (d) Amplitude vs frequency curves before and after a mass is added to cantilever 2 in (a). The relative changes in the eigenstates upon addition of the mass are 5\% and 7\% while the relative changes in resonance frequencies are $\sim 0.01 \%$, well within the natural drift of the sensor under ambient conditions. Images of the finite element in-phase and out-of-phase eigenstates are also shown (inset). The response curves are obtained by first measuring the response of cantilever 1 and then the response of cantilever 2 both before and after mass is added.

eigenstates still remains more than one order of magnitude higher than the relative change of the eigenvalue (resonance frequency) of a single cantilever.

The coupled gold-foil cantilevers are produced by first bonding a piece of gold foil, obtained from Goodfellow Inc.,
to AlP license or copyright, see http://apl.aip.org/apl/copyright.jsp 


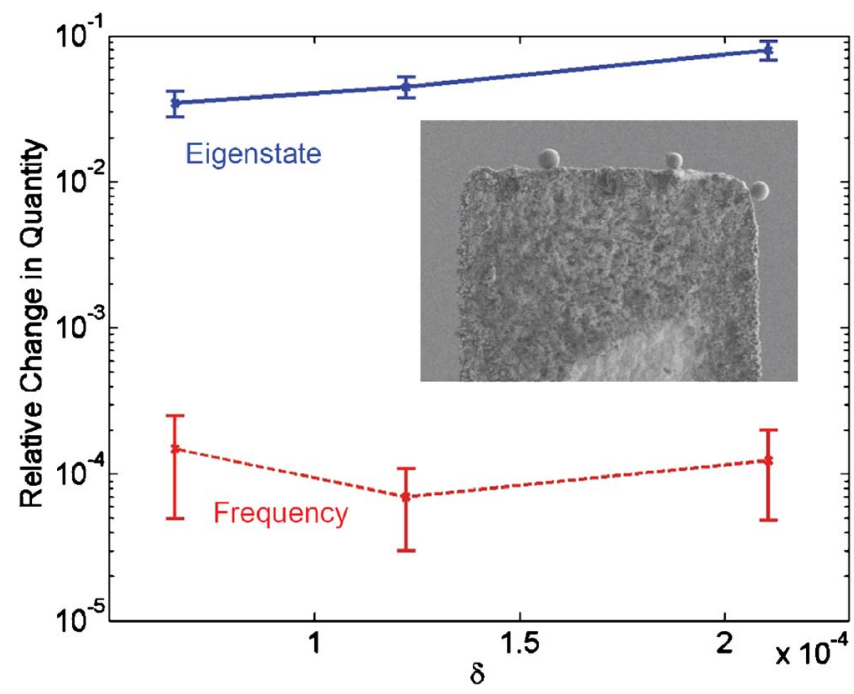

FIG. 3. (Color online) Relative change in in-phase eigenstate (solid line) and relative change in frequency (dashed line) vs amount of nondimensional mass $\delta$ added to cantilever 2 (shown in the inset with 3 attached microspheres) for the second set of coupled gold-foil cantilevers tested. The second set had less initial disorder than the first set; therefore, only the in-phase eigenstate was observed before mass loading.

to a silicon base. The cantilevers and overhang, shown in Fig. 2(a), are machined from the foil using an amplified Ti:sapphire femtosecond laser. The results for two sets of coupled gold-foil cantilevers are discussed below. The cantilever vibrations are analyzed in a Nanotec Electronica ${ }^{\mathrm{TM}}$ scanning probe microscopy system. Frequency sweeps are performed at $1 \mathrm{~Hz}$ intervals on both cantilevers separately to obtain a fine frequency response curve.

Frequency sweeps of the first set of coupled gold-foil cantilevers tested show two clear peaks corresponding to the in-phase and out-of-phase eigenstates, as shown in Fig. 2(b). Examining the phase data in Fig. 2(c) confirms that the cantilevers vibrate in the in-phase eigenstate at the lower resonance peak, and in the out-of-phase eigenstate at the higher resonance peak. Since the resonance peaks do not overlap, each eigenstate is simply the normalized vector formed by the amplitudes of the two cantilevers at a resonance frequency.

After measurement of the initial eigenstates, borosilicate microspheres from Duke Scientific (mean diameter of $4.9 \mu \mathrm{m}$ with a mass of $\sim 154 \mathrm{pg}$ ) were placed on one cantilever. Adding the mass to cantilever 2 in Fig. 2(a) produces significant changes in the eigenstates $(5 \%-7 \%)$ of the first set of coupled gold-foil cantilevers tested, as shown in Fig. 2(d). As expected, the relative change in the resonance frequency is $\sim 0.01 \%$, a value well within the range of natural drift of resonance frequencies for this gold-foil sensor. Removing the mass caused the eigenstates of both sensors to return to near their original values.

The effect of adding multiple masses was also studied using a second set of coupled gold-foil cantilevers as shown in Fig. 3, where the relative change in the eigenstates and resonance frequencies is plotted. As predicted, the measured relative change of the in-phase eigenstate is more than two orders of magnitude greater than the relative change in its resonance frequency.

The mode localization sensor concept possesses several particularly attractive features. First, its high sensitivity is relatively independent of the quality factors of resonance. Second, as evident from Eqs. (1) and (4) this sensor possesses intrinsic common mode rejection since the eigenstates are insensitive to environmental drifts common to both cantilevers; instead this sensor naturally detects differences in added mass or stiffness between the two cantilevers. Both attributes are crucial for sensing under ambient conditions. Finally, additional calculations show that the observed sensitivity to added mass can be extended to large arrays of coupled microcantilevers, thereby allowing this sensing paradigm to be used to sense multiple analytes with high sensitivity using appropriately functionalized cantilevers.

In conclusion, we have achieved two orders of magnitude improvement in sensitivity under ambient conditions over the conventional frequency shift based sensors. These results are achieved without any particular attempt at optimizing the design of the coupled microcantilevers. With further optimization we expect that the sensitivity of such mode localization based sensors could be three to four orders of magnitude greater than for frequency shift based sensors.

This work was partially supported by NASA Grant No. NCC 2-1363.

${ }^{1}$ B. Rogers, L. Manning, M. Jones, T. Sulchek, K. Murray, B. Beneschott, J. D. Adams, Z. Hu, T. Thundat, H. Cavazos, and S. C. Minne, Rev. Sci. Instrum. 74, 4899 (2003).

${ }^{2}$ F. M. Battiston, J.-P. Ramseyer, H. P. Lang, M. K. Baller, Ch. Gerber, J. K. Gimzewski, and H.-J. Güntherodt, Sens. Actuators B 77, 122 (2001).

${ }^{3}$ L. A. Pinnaduwage, A. Wig, D. L. Hedden, A. Gehl, D. Yi, T. Thundat, and R. T. Lareau, J. Appl. Phys. 95, 5871 (2004).

${ }^{4}$ A. Gupta, D. Akin, and R. Bashir, Appl. Phys. Lett. 84, 1976 (2004).

${ }^{5}$ K. L. Ekinci, X. M. H. Huang, and M. L. Roukes, Appl. Phys. Lett. 84, 4469 (2004).

${ }^{6}$ T. Ono, X. Li, H. Miyashita, and M. Esashi, Rev. Sci. Instrum. 74, 1240 (2003).

${ }^{7}$ Z. Davis and A. Boisen, Appl. Phys. Lett. 87, 013102 (2005).

${ }^{8}$ H. Sone, Y. Fujinuma, and S. Hosaka, Jpn. J. Appl. Phys., Part 1 43, 3648 (2004).

${ }^{9}$ B. Ilic, D. Czaplewski, M. Zalalutdinov, and H. G. Craighead, J. Vac. Sci. Technol. B 19, 2825 (2001).

${ }^{10}$ P. W. Anderson, Phys. Rev. 109, 1492 (1958).

${ }^{11}$ C. Pierre, J. Sound Vib. 126, 485 (1988).

${ }^{12}$ C. Pierre and E. H. Dowell, J. Sound Vib. 114, 549 (1987).

${ }^{13}$ C. Pierre, D. M. Tang, and E. H. Dowell, AIAA J. 25, 1249 (1987).

${ }^{14}$ P.-T. Chen and J. H. Ginsburg, J. Vibr. Acoust. 114, 141 (1992).

${ }^{15}$ O. O. Bendiksen, AIAA J. 25, 1241 (1987).

${ }^{16}$ M. Sato, B. E. Hubbard, A. J. Sievers, B. Ilic, D. A. Czaplewski, and H. G. Craighead, Phys. Rev. Lett. 90, 044102 (2003).

${ }^{17}$ E. Buks and M. L. Roukes, J. Microelectromech. Syst. 11, 802 (2002).

${ }^{18}$ M. Napoli, W. Zhang, K. Turner, and B. Bamieh, J. Microelectromech. Syst. 14, 295 (2005).

${ }^{19}$ R. Courant and D. Hilbert, Methods of Mathematical Physics (Interscience, New York, 1953), Vol. 1.

${ }^{20}$ The nominal length, width, and thickness of each microcantilever are $500 \mu \mathrm{m}, 100 \mu \mathrm{m}$, and $10 \mu \mathrm{m}$, respectively. The cantilevers are separated by $250 \mu \mathrm{m}$; the length of the overhang is $90 \mu \mathrm{m}$. The mass of an individual gold-foil cantilever is $\sim 9.5 \mu \mathrm{g}$ and it has a resonance frequency near $13.4 \mathrm{kHz}$. The length, width, and thickness of both cantilevers are varied using a zero-average normal distribution with standard deviations of $0.5 \mu \mathrm{m}, 0.5 \mu \mathrm{m}$, and $0.1 \mu \mathrm{m}$, respectively. These values represent the accuracy of the machining process and the likely thickness variation that occurs in the metal foil. 